\title{
3 Research Square \\ Exosomal miR-132-3p from Mesenchymal Stromal Cells Improve Synaptic Dysfunction and Cognitive Decline in Vascular Dementia
}

\section{Xiaotang Ma}

affiliated hospital of Guangdong medical university

\section{Yan Wang}

affiliated hospital of guangdong medical university

\section{Yumeng Shi}

Affiliated hospital of guangdong medical university

\section{Suqing Li}

affiliated hospital of guangdong medical university

Jinhua Liu

affiliated hospital of guangdong medical university

\section{Xiangyong Li}

Guangdong medical university

\section{Wangtao Zhong}

affiliated hospital of guangdong medical university

\section{Qunwen Pan ( $\nabla$ pqwforeve@163.com )}

Guangdong key laboratory of age-related cardiac and cerebral diseases, Institute of Neurology https://orcid.org/0000-0002-8705-3062

\section{Research Article}

Keywords: vascular dementia, mesenchymal stromal cells, exosomes, miR-132-3p, synaptic plasticity.

Posted Date: December 10th, 2021

DOI: https://doi.org/10.21203/rs.3.rs-1048848/v1

License: (c) (1) This work is licensed under a Creative Commons Attribution 4.0 International License.

Read Full License 


\section{Abstract}

Background/Aims: Vascular dementia (VD) results in cognition and memory deficit. Exosomes and their carried microRNAs (miRs) contribute to the neuroprotective effects of mesenchymal stromal cells, and miR-132-3p plays a key role in neuron plasticity. Here we investigated the role and underlying mechanism of MSC EX and their miR-132-3p cargo in rescuing cognition and memory deficit in VD mice.

Methods: Bilateral carotid artery occlusion was used to generate a VD mouse model. MiR-132-3p and MSC EX levels in the hippocampus and cortex were measured. At $24 \mathrm{~h}$ post-VD induction, mice were administered with MSC EX infected with control lentivirus (EX $\left.{ }^{\mathrm{Con}}\right)$, pre-miR-132-3p-expressing lentivirus (EX $\left.{ }^{m i R-132-3 p}\right)$, or miR-132-3p antago lentivirus (EXantagomiR-132-3p) intravenously. Behavioral and cognitive tests were performed and the mice were sacrificed in 21 days after VD. The effects of MSC EX on neuron number, synaptic plasticity, dendritic spine density, and $A \beta$ and $p$-Tau levels in the hippocampus and cortex were determined. The effects of MSC EX on oxygen-glucose deprivation (OGD)injured neurons with respect to apoptosis, and neurite elongation and branching were determined. Finally, the expression levels of Ras, phosphorylation of Akt, GSK-3 $\beta$, and Tau were also measured.

Results: Compared with normal mice, VD mice exhibited significantly decreased miR-132-3p and MSC EX levels in the cortex and hippocampus. Compared with EX ${ }^{\text {Con }}$ treatment, the infusion of EX ${ }^{m i R-132-3 p}$ was more effective at improving cognitive function and increasing miR-132-3p level, neuron number, synaptic plasticity, and dendritic spine density, while decreasing $A \beta$ and $p$-Tau levels in the cortex and hippocampus of VD mice. Conversely, EXantagomiR-132-3p treatment significantly decreased miR-132-3p expression in cortex and hippocampus, as well as attenuated EX ${ }^{\text {miR-132-3p }}$ treatment-induced functional improvement. In vitro, EX ${ }^{\text {miR-132-3p }}$ treatment inhibited RASA1 protein expression, but increased Ras and the phosphorylation of Akt and GSK-3 3 , and decreased p-Tau levels in primary neurons by delivering miR132-3p, which resulted in reduced apoptosis, and increased neurite elongation and branching in OGDinjured neurons.

Conclusions: Our studies suggest that miR-132-3p cluster-enriched MSC EX promotes the recovery of cognitive function by improving neuronal and synaptic dysfunction through activation of the Ras/Akt/GSK-3ß pathway induced by downregulation of RASA1.

\section{Introduction}

Vascular dementia (VD), a degenerative cerebrovascular disorder resulting in cognitive impairment and memory decline,is currently the second leading form of dementia (at least $20 \%$ ) following Alzheimer's disease (AD) $\square$ However, there are still no available treatments to slow or reverse the damage caused by $V D[1]$. Similar to $A D$ and other cognitive disorders, neuronal damage and synaptic dysfunction in the hippocampus and the cortex are the main causes of VD [1]. Therefore, protection of neuronal density, synapse formation, and synaptic plasticity is important for ameliorating cognitive impairment after VD. 
Mesenchymal stromal cells (MSC), which can be conveniently isolated from various tissues, have shown potential therapeutic effects on neurological diseases and injury through neuronal differentiation and paracrine action[2, 3]. Recent studies suggested that the paracrine activity, rather than differentiation, played a vital role in the protective effects of MSCs $[4,5]$. Exosomes (EX), a nanosized membrane vesicles secreted by most cells, may affect recipient cell function by transferring their cargos, including protein and microRNAs (miRs), which are considered a novel tool for stem cell paracrine action[6]. Several studies had demonstrated that EXs derived from MSC (MSC EX) exerted therapeutic effects on neural impairment in various neurological diseases, such as ischemic stroke and $A D[6,7]$. In an $A D$ mouse model, systemic administration of MSC EX ameliorated cognitive decline by decreasing A $\beta$ accumulation and synaptic dysfunction [8]. However, there are no reports demonstrating whether MSC EX can protect neuronal damage and cognitive decline resulting from VD.

Studies have shown that the therapeutic effects of MSC EX are highly correlated with their miR cargos. Therefore, the use of EXs engineered to carry neuronal protective miRs for the treatment of neurological diseases represents a promising strategy. Chopp et al. reported that miR-17-enriched MSC EX improved neurological outcome by increasing neurogenesis and neurite outcome in a rat stroke model[9]. MiR-132$3 p$ is one of the most abundant miRs in brain tissue and has been shown to exert a strong neuroprotective effect[10]. In the hippocampus, a reduction of miR-132-3p was associated with decreased spine density, reduced synapse formation, increased $A \beta$ production, and Tau hyperphosphorylation [11, 12]. In primary cultured neurons, miR-132-3p inhibition induced cell apoptosis via the PTEN/Akt signaling pathway. Furthermore, studies had shown that miR-132 promoted axon outgrowth by repressing the targeted gene, Rasa1, and activating the Ras signaling pathway[13, 14]. The Ras/Akt/GSK-3 $\beta$ signaling pathway has been found to be implicated in the regulation of axonal outgrowth and neuronal function recovery [15]. Thus, we hypothesize that enrichment of miR-132-3p may increase the beneficial effects of MSC EX on VD-induced neuron damage and cognitive impairment.

In this study, we investigated the effects of miR-132-3p-enriched MSC EX on neuron damage, synaptic dysfunction, and cognitive impairment in a VD mouse model and the underlying mechanism of action by analyzing the Ras/Akt/GSK-3 $\beta$ signaling pathway.

\section{Materials And Methods}

\section{Generation of miR-132-3p enriched or knock down MSC exosomes}

Mesenchymal stem cells (MSC) were cultured from bone marrow of C57BL/6 mice as we previously described [16]. Control MSC exosomes (EX $\left.{ }^{\mathrm{Con}}\right)$, miR-132-3p enriched MSC exosomes (EX ${ }^{\text {miR-132-3p }}$ ), and miR-132-3p knock down MSC exosomes (EX antagomiR-132-3p $)$ were collected from MSC transfected with scrambled control (MSC $\left.{ }^{\mathrm{Con}}\right)$, lentivirus carrying the murine miR-132-3p (MSC ${ }^{\text {miR-132-3p }}$ ), or levtivirus

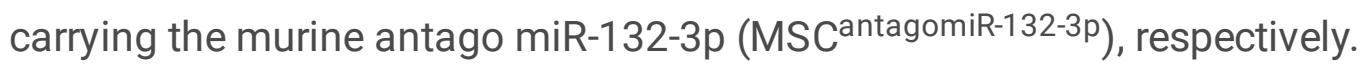

\section{Animals}


Adult (8-12 weeks old) male C57BL/6 mice were purchased from the Animal Experiment Center of Guangdong Province (Guangzhou, China) and raised in the animal care facility at the Guangdong Medical University. The mice were housed in a pathogen-free environment. Surgeries were performed under $2.5 \%$ isoflurane anesthesia. All experimental procedures were approved by the laboratory animal care and use committees at Guangdong Medical University.

\section{EX infusion of mouse VD model}

The VD mouse model was generated as previously described [17]. Briefly, mice were anesthetized with isoflurane, fixed on a stereotaxic instrument, and body temperature was maintained at $37^{\circ} \mathrm{C}$ with a feedback-regulated water heating system. A skin incision was made in the neck area, the bilateral carotid arteries were separated and locked by ligation for $10 \mathrm{~min}$ and then released for $10 \mathrm{~min}$. This process was repeated three times. The sham-operated mice (Control) underwent the same procedure except the bilateral carotid arteries were locked. Twenty-four hours after VD, the mice were administered PBS

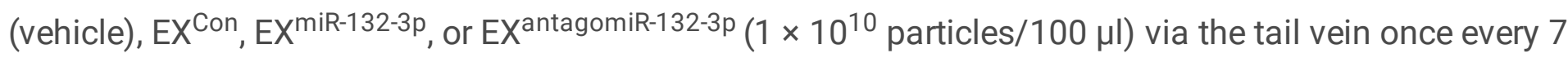
days for 21 days. This dose was selected based on previous studies on MSC EX administration for treating CNS diseases[18]. Memory and cognitive performance were measured by the Morris water maze (MWM) test on the last 5 days of the experiment. Immediately after the MWM test, the mice were sacrificed at day 21 following MSC EX infusion, and the cortex and hippocampus were collected and used for various measurements including NTA analysis, immunofluorescence, immunohistochemistry, and Golgi staining.

\section{Detection of MSC EX in brain tissue}

MSC EX were isolated from the cortex and hippocampus of Sham (MSC EX ${ }^{\text {Sham }}$ ) or VD (MSC EXVD) mice as described previously with some modification[19]. Briefly, the cortex and hippocampus were separated and finely minced with small, sharp scissors in $100 \mu \mathrm{l}$ of papain solution (20 units/ml). Brains in solution were pipetted into a $15 \mathrm{ml}$ conical tube containing $3.5 \mathrm{ml}$ papain solution and incubated for $20 \mathrm{~min}$ at $37^{\circ} \mathrm{C}$ to dissociate the tissue and free the extracellular space. Then, the total brain EXs were isolated using sequential centrifugation at $300 \mathrm{xg}$ for $10 \mathrm{~min}, 2000 \mathrm{x} \mathrm{g}$ for $20 \mathrm{~min}, 20,000 \mathrm{x} \mathrm{g}$ for $90 \mathrm{~min}$, and $100,000 \times \mathrm{g}$ for $2 \mathrm{~h}$ after the supernatant was passed through a $0.22 \mu \mathrm{m}$ syringe filter (Millex-GP, Millipore). The pelleted EXs were incubated with $10 \mu$ of biotin-conjugated anti-CD29 antibody (Miltenyi Biotec) in a $100 \mu \mathrm{l}$ reaction volume for $2 \mathrm{~h}$, followed by the addition of $10 \mu \mathrm{l}$ of anti-biotin microbeads for $15 \mathrm{~min}$. The microbeads labeled EX from the total brain EX suspension were separated with a DynaMag-2 magnet (Life technology). After an overnight magnetic separation, the microbead-bound EXs were collected and resuspended in $100 \mu \mathrm{l}$ of particle-free PBS. The suspension was incubated with $10 \mu \mathrm{l}$ multisort release reagent (Miltenyi Biotec) to cleave off the microbeads. The EX in the fluid were collected and considered MSC EX (CD29+ EXs). The pelleted MSC EX were resuspended in phosphate-buffered saline (PBS) and aliquoted for nanoparticle tract analysis (NTA), transmission electron microscopy, and EX-specific marker CD63 and MSC-specific marker CD29 measurement using western blot analysis. 


\section{Detection of MSC EX interacting with neurons in the cortex and hippocampus}

MSC EX were labeled with PKH26 (Sigma) and resuspended in PBS for infusion [8]. After $24 \mathrm{~h}$ of infusion, the brains were dissected from mice, frozen in liquid nitrogen, and cut into $20-\mu \mathrm{m}$-thick sections. The brain sections were incubated with rabbit monoclonal anti-NeuN antibody $(1: 300, \mathrm{Abcam})$ at $4^{\circ} \mathrm{C}$ overnight and the sections were then incubated with goat anti-rabbit IgG H\&L (Alexa Fluor ${ }^{\circledR} 488$ ) secondary antibody for $1 \mathrm{~h}$. After rinsing with wash solution, the interaction of MSC EX with neurons in the cortex and hippocampus were detected by confocal microscopy (Olympus Corporation, Japan).

\section{Quantitative RT-PCR}

The levels of miR-132-3p in MSC, MSC EX, neurons, cortices, and the hippocampus were measured by quantitative real-time PCR (qRT-PCR). Total miRs from mice and cells were isolated using the miRNeasy Mini kit (QIAGEN) based on the manufacturer's instructions and miR-132-3p cDNA was generated for qRTPCR analysis (Roche 480). Samples were analyzed in triplicate and U6 was used as an internal control. The primer sequences used are listed below: 5-CCAGCATAACAGTCTACAGCCA-3 and 5AACGCTTCACGAATTTGCGT-3 for miR-132-3p, and 5-CTCGCTTCGGCAGCACA-3 and 5AACGCTTCACGAATTTGCGT-3 for U6. A total reaction mixture of $10 \mu \mathrm{l}$ was amplified in a 96-well PCR plate (BIOplastics, Netherlands) using the following cycling conditions: $95^{\circ} \mathrm{C}$ for 3 min followed by 40 cycles of $95^{\circ} \mathrm{C}$ for $12 \mathrm{~s}$ and $60^{\circ} \mathrm{C}$ for $40 \mathrm{~s}$.

\section{Morris water maze test}

The labyrinth was composed of a barrel water tank $(120 \mathrm{~cm}$ in diameter with a platform filled with tap water at a temperature of $22 \pm 2^{\circ} \mathrm{C}$ ). An escape platform with a diameter of $10 \mathrm{~cm}$ was placed $1-2 \mathrm{~cm}$ below the water surface, mice were place into the maze at one of four points $(N, S, E, W)$ facing the wall of the tank. Over a period of five days, the mice were allowed to find the platform for $90 \mathrm{~s}$. If the mice failed to find the platform, it was guided to the platform and allowed to rest for $15 \mathrm{~s}$. On day five, the platform was removed and a probe test was conducted. The percentage time spent in each of the four quadrants and the number of target area crossings, total distance, and mean speed were recorded.

\section{Immunohistochemistry}

The number and arrangement of neurons in hippocampal CA1 region were detected by immunohistochemical staining. Each group of brain tissue was fixed in $4 \%$ formalin for $24 \mathrm{~h}$, and then the samples were dehydrated in serially graded ethanol solutions, defatted in methanol and embedded in paraffin. The brain tissues were coronally sectioned at a thickness of $6 \mu \mathrm{m}$, and deparaffinized in xylene, rehydrated in descending concentrations of alcohol. Endogenous peroxidase activity was blocked with $3 \% \mathrm{H}_{2} \mathrm{O}_{2}(\mathrm{v} / \mathrm{v})$ for $20 \mathrm{~min}$. The sections were placed in $0.01 \mathrm{M}$ citrate buffer and heated in a microwave oven at $95^{\circ} \mathrm{C}$ for $20 \mathrm{~min}$ after washed with PBS. Brain sections were stained with hematoxylin and eosin (H\&E), followed by dehydration, hyalinization, fixation, and observed under a high-magnification optical microscope (Olympus, Japan). Neuronal damage was evaluated on a scale of $0=$ normal, $1=$ a few $(<$ 
$30 \%$ ) neurons damaged, 2 = many (30 to $70 \%$ ) neurons damaged, and $3=$ majority of neurons ( $70 \%)$ damaged (ref: Strain related differences in susceptibility to transient forebrain ischemia in SV-129 and C57 Black/6 mice).

\section{Immunofluorescence staining}

Mouse brain sections or cultured neurons were fixed in $4 \%$ paraformaldehyde and washed with PBS three times. Antigen retrieval was performed with citrate buffer $(\mathrm{pH} 7.0)$. The samples were then permeabilized with $0.1 \%$ Triton X-100 for 5 min on ice, blocked in PBS containing $10 \%$ normal goat serum at room temperature for one hour, and washed three times with PBS. Brain sections and cultured neurons were incubated with primary antibodies overnight at $4^{\circ} \mathrm{C}$. After washing with PBS, the samples were incubated with secondary antibodies in blocking buffer for $1 \mathrm{~h}$. Subsequently, the cellular nucleus was stained with DAPI (1:1000, Abcam) for $7 \mathrm{~min}$ at room temperature. Samples were washed with wash buffer three times and images were acquired using a confocal microscope (Olympus, Japan).

The primary antibodies used for immunostaining included NeuN (rabbit, 1:300, Abcam), MAP2 (rabbit, 1:500, Abcam), Tau46 (mouse, 1:200, Millipore), A (rabbit, 1:300, Abcam), PSD95 (1:500, Abcam), and synaptophysin (1:500, Abcam). The samples were then incubated with 488/564/647 goat antirabbit/mouse secondary antibodies (1:250, Invitrogen).

\section{Golgi staining}

Golgi staining was performed using the FD Rapid Golgi Stain system (FD Neuro Technologies) according to the manufacturer's instructions. Briefly, freshly dissected brains were immersed in the impregnation solution and stored at room temperature in the dark for two weeks. Brains were then transferred to solution $\mathrm{C}$ and stored at $4^{\circ} \mathrm{C}$ for $48 \mathrm{~h}$ in the dark. Brains were coronally sectioned at a thickness of 200 $\mu \mathrm{m}$ with a freezing microtome (Thermo Scientific, USA). Brain sections were stained with solution D and E. Golgi-stained neurons and dendritic segments in the cortex and hippocampal regions were observed using an Olympus confocal microscope. Dendritic branching and spine density was detected using NIH Image $\mathrm{J}$ software.

\section{Neuron OGD model and functional analysis}

\section{Isolation of primary neurons}

Primary neurons were cultured from C57BL/ 6 mouse embryos (aged 5-8 days). Briefly, brain tissues were dissected from mouse pups and the meninges were removed from the brain tissue and dissociated by enzymatic digestion. Isolated primary neurons were plated onto poly-D-lysine-coated dishes and cultured in Neurobasal medium supplemented with $2 \%$ B27 (Gibco), $0.5 \mathrm{mM}$ of glutamine, $10 \%$ fetal bovine serum (Gibco), and $1 \%$ penicillin/streptomycin (Sigma) and maintained in a $5 \% \mathrm{CO}_{2}$ incubator at $37^{\circ} \mathrm{C}$. The media was changed every three days. 
The neuron oxygen-glucose deprivation (OGD) model was established according to a previously publication with minor modification[20]. Briefly, the cultured primary neurons were washed and incubated with glucose-free DMEM (Gibco, USA) and incubated in an anaerobic chamber (Thermo Fisher Scientific, USA) containing $1 \% \mathrm{O}_{2}, 5 \% \mathrm{CO}_{2}$, and $94 \% \mathrm{~N}_{2}$ for $2 \mathrm{~h}$. After incubation, the culture medium was replaced with normal medium in a normoxic condition. During reoxygenation time, neurons were co-cultured with various MSC EX (EXCon, EX ${ }^{\text {miR-132-3p }}$ ) or culture medium (vehicle). To explore the underlying mechanism, neurons were pretreated with a Ras inhibitor (NSC 23766, 100 mM, Sellectkchem) for 2 h.

\section{Neuron functional analysis}

Neuron viability was measured using the CCK-8 assay kit according to the manufacturer's instructions. Neuronal apoptosis was analyzed using the Annexin V-PE/7-AAD apoptosis detection kit (BD Bioscience, USA) based on the manufacturer's instructions followed by flow cytometric analysis. Intracellular ROS production by neurons was measured by dihydroenthidium (DHE, Beyontime, China) staining and visualized under a confocal microscope (Olympus, Japan).

\section{Western blot analyses}

The neurons were homogenized in lysis buffer including proteinase and phosphate kinase inhibitors (Sigma, USA). Proteins $(30 \mu \mathrm{g})$ were mixed with loading buffer and electrophoresed through SDS-PAGE gels and transferred to polyvinylidene difluoride membranes (PVDF, Millipore Corporation, USA). After blocking non-specific antigens in $5 \%$ non-fat milk, the membranes were incubated with primary antibodies against RASA1 (1:1000, CST, USA), Ras (1:1000, Abcam, USA), Akt and phospho-Akt (1:500, CST), GSK-3 $\beta$ and phospho-GSK-3 $\beta^{\text {ser9 }}$ (1:1000, Abcam), p-Tau (1:1000, Abcam), and CD63 (1:400, Abcam). The bands were visualized using an ECL kit (Amersham, Sweden).

\section{Results}

\section{Infusion of EXmiR-132-3p can restore cortical and hippocampal MSC EX and miR-132-3p in VD mice}

NTA and TEM analyses showed that the MSC EX Sham and MSC EXVD were $100 \pm 50 \mathrm{~nm}$ in size (Fig. 1A, 1B). By using western blotting assay, we verified that both of the MSC EXSham and MSC EXVD were positive for CD63 and CD29 (Fig. 1C). These data indicating MSC EX were successfully isolated from mouse brain. Twenty-one days following VD surgery, cortical and hippocampal MSC EX levels were detected by combining microbeads with NTA. The level of the MSC EX was decreased by $78 \%$ in the cortex and $85 \%$ in the hippocampus of VD mice at 21 days following VD surgery compared with that of the sham group (vs sham; $p<0.05$; Fig. 1D). qRT-PCR analysis revealed that the level of miR-132-3p was decreased $72.9 \%$ in the cortex and $75.6 \%$ in the hippocampus at 21 days after VD surgery, compared with that of the sham group (vs sham group; $p<0.05$; Fig. 1E). These data suggest that the reduction of miR132-3p and MSC EX in the cortex and hippocampus was highly correlated with VD injury. 


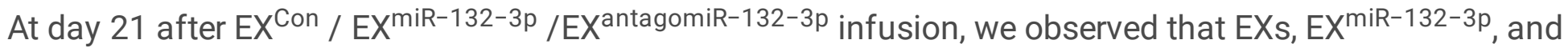
EXs ${ }^{\text {antagomiR-132-3p }}$ significantly increased MSC EX levels in the cortex and hippocampus of VD mice (vs Sham, $p<0.05$; Fig. 1E). Meanwhile, we also observed that there were no significant differences between the three treatments (EX ${ }^{C o n}$ vs EX ${ }^{m i R-132-3 p}$ or EX ${ }^{m i R-132-3 p}$ vs EX antagomiR-132-3p,$p>0.05$; Fig. 1E). Using qRT-PCR analysis, we found that both EX and EX ${ }^{m i R-132-3 p}$ increased miR-132-3p levels in the cortex and hippocampus of VD mice (vs PBS, $p<0.05$; Fig. 1E) and EX ${ }^{m i R-132-3 p}$ displayed better efficacy at increasing miR-132-3p levels compared with EX-treatment (vs EX, $p<0.05$; Fig. 1E) and EXantagomiR-132-3p decreased miR-132-3p levels compared with EXmiR-132-3p (vs EXmiR-132-3p,$p<0.05$; Fig. 1E). These results suggest that $\mathrm{EX}^{\mathrm{Con}} / \mathrm{EX}^{\mathrm{miR}-132-3 \mathrm{p}}$ / EX antagomiR-132-3p infusion can restore attenuated MSC EX levels in the cortex and hippocampus of VD mice, and EX ${ }^{\text {miR-132-3p }}$ can increase cortical and hippocampal miR-132-3p via transferring their contained miR-132-3p.

\section{MiR-132-3p mediates EX-induced restoration of cognitive and synaptic functions in VD mice}

As shown in Fig. 2B, we confirmed the fluorescent of PKH26-labeled MSC EX was observed in cortical and hippocampal neurons in VD mice, suggesting that the injected MSC EX merged into the cortical and hippocampal neurons. To test whether MSC EX and their carried miR-132-3p could rescue synaptic deficits, learning, and memory impairment in VD mice, EXCon /EXmiR-132-3p/ EX antagomiR-132-3p were injected into VD mice intravenously every 7 days for 21 days. We observed that both EX and EXmiR-132-3p rescued escape latency deficits during training and improved time spent in the target quadrant and numbers to cross the platform in MWM tests (vs PBS, $p<0.05$; Fig. 2C-2F), with a better effect observed with $\mathrm{EX}^{\mathrm{miR}-132-3 \mathrm{p}}$ (vs EXCon, $\mathrm{p}<0.05$; Fig. 2C-2F). Whereas EXantagomiR-132-3p decreased cognitive function restoration compared with EX $X^{m i R-132-3 p}$-treated group (vs EXmiR-132-3p, $p<0.05$; Fig. 2C-2F). In addition, synaptic spines and dendritic complexity compromised in cortex and hippocampus of VD mice were also restored by EXCon infusion (vs PBS, $p<0.05$; Fig. 3A-3E). Similarly, infusion of EX ${ }^{\text {miR-132-3p }}$ significantly induced an increase of synaptic spines and dendritic complexity compared with EX-treated group (vs EXCon, $p<0.05$; Fig. 3A-3E) and EX antagomiR-132-3p decreased cortical and hippocampal synaptic spines and dendritic complexity compared with EX ${ }^{m i R-132-3 p}$ (vs EX miR-132-3p $^{\text {p }}<0.05$; Fig. 3A$3 E$ ). These data indicated that miR-132-3p enrichment enhances the effects of EX on restoring cognitive and synaptic functions in VD mice.

\section{MiR-132-3p mediates MSC EX-induced reduction of cortical and hippocampal neuronal damage, $A \beta$ production, and Tau hyperphosphorylation in VD mice}


To test the effects of MSC EX and their carried miR-132-3pon cortical and hippocampal neuron damage in VD mice, immunochemistry and immunofluorescence were performed in brain sections day 21 after carotid artery ligation. Compared with the sham group, VD mice showed evident neuronal cell loss and abnormal architecture in the hippocampal CA1 region, concomitant with increased neuronal cell apoptosis in the cortex (vs sham, $p<0.05$; Fig. 4A-4D). EX ${ }^{\text {Con }}$ and EX ${ }^{m i R-132-3 p}$ infusion significantly reduced neuronal cell loss and abnormal architecture in the hippocampal CA1 region in VD mice (vs PBS, $p<0.05$; Fig. 4A-4D). To our expectation, EX ${ }^{m i R-132-3 p}$ displayed better efficacy on reducing neuronal cell loss and abnormal architecture compared with EX-treated group (vs EXCon,$p<0.05$; Fig. 4A-4D) and $E X^{\text {antagomiR-132-3p }}$ showed attenuated effect on reducing cortical and hippocampal neuron damage (vs EX $X^{m i R-132-3 p}, p<0.05$; Fig. 4A-4D). Additionally, EXCon infusion significantly decreased cortical neuron

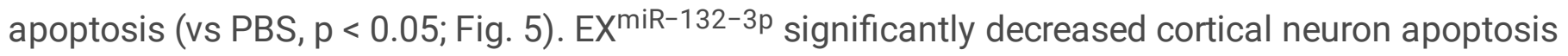
compared with EXCon (vs EXCon, $p<0.05$; Fig. 5) and EXantagomiR-132-3p showed attenuated effect on decreasing cortical neuron apoptosis (vs EXmiR-132-3p,$p<0.05$; Fig. 5).

As shown in Fig. 6 and Fig. 7, A $\beta$ production and Tau phosphorylation in the cortex and hippocampus were increased in VD mice (vs sham, $p<0.05$ ). According to our "treatment" study, we observed that

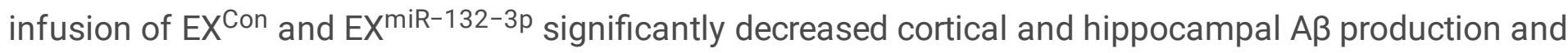
Tau hyperphosphorylation (vs Sham, p < 0.05; Fig. 6 and Fig. 7). Similarly, EX ${ }^{\text {miR-132-3p }}$ were more effective than $E X$ in reducing $A \beta$ production and Tau phosphorylation compared with $E X^{\text {miR-132-3p }}$ and $E X^{\text {antagomiR-132-3p }}$ showed attenuated effect on reducing $A \beta$ production and Tau phosphorylation (vs EX ${ }^{\text {miR-132-3p }}$, p $<0.05$; Fig. 6 and Fig. 7).

Taken together, these results suggest that miR-132-3p enrichment enhances the effects of MSC EX on reducing neuronal cell damage, $A \beta$ overproduction, and Tau hyperphosphorylation in the cortex and hippocampus of VD mice.

\section{MiR-132-3p mediates MSC EX-induced activation of the Ras/Akt/- GSK-3 $\beta$ signaling pathway in OGD-injured neurons}

After co-culture for $24 \mathrm{~h}, \mathrm{PKH} 26$-labeled MSC EX was detected in the cytoplasm of primary neurons (Fig. 8A), suggesting that EX had merged with the neurons. Using qRT-PCR, we found that EXCon and $E X^{m i R-132-3 p}$ incubation significantly increased miR-132-3p expression in recipient neurons (vs vehicle, $p$ $<0.05$; Fig. 8B). EX ${ }^{m i R-132-3 p}$ were more effective at increasing miR-132-3p levels compared with EXCon

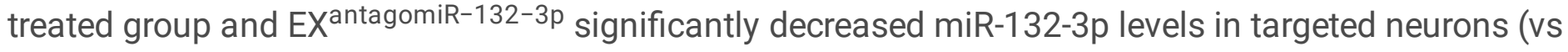
EXmiR-132-3p,$p<0.05$; Fig. 8B). These data indicate that miR-132-3p can be transferred from MSC to neurons by $E^{m i R-132-3 p}$.

Using bioinformatics analysis, we found RASA1 is a predicted target of miR-132-3p (Fig. 8C). As expected, we found that RASA1 expression in OGD-treated neurons was significantly decreased after 
EXCon incubation (vs vehicle, $p<0.05$; Fig. $8 C$ ) and $E X^{m i R-132-3 p}$ exhibit greater effects on decreasing

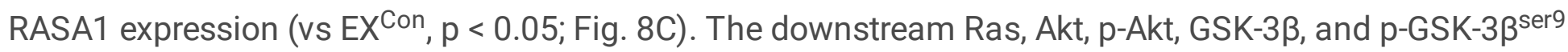
proteins levels were also examined by western blotting assay. We found that OGD treatment significantly decreased Ras expression and the phosphorylation of Akt and GSK-3 $\beta$ in neurons (vs control, $p<0.05$; Fig. 9A, 9B).. EX incubation increased Ras and the phosphorylation of Akt and GSK-3 $\beta$ in OGD-treated neurons (vs vehicle, $p<0.05$; Fig. 9A, 9B). Again, $E X^{m i R-132-3 p}$ were more effective in increasing Ras and the phosphorylation of Akt and GSK-3 $\beta$ compared with EX ${ }^{\text {Con }}$-treated group and Ras inhibitor (NSC23766) partially abolished the effects of EX ${ }^{\mathrm{miR}-132-3 p}$ on increasing Ras levels and phosphorylation of Akt and GSK-3 $\beta$ in OGD-treated neurons (vs EX ${ }^{\text {miR-132-3p }}, p<0.05$; Fig. 9A, 9B). These results suggest that miR132-3p enrichment enhances the effects of MSC EX on activating the Ras/Akt/GSK-3 $\beta$ signaling pathway by targeting RASA1 in OGD-treated neurons.

\section{MiR-132-3p mediates EX-induced decreasing of Tau hyperphosphorylation, and apoptosis in OGD-injured neurons by activating the Ras/Akt/-GSK-3 $\beta$ signaling pathway}

As shown in Fig. 9C-9F, we observed that OGD-treated neurons exhibited a marked increase in Tau phosphorylation, and apoptosis (vs sham, $\mathrm{p}<0.05$ ), and EXCon incubation decreased Tau phosphorylation and apoptosis in OGD-treated neurons (vs vehicle, $p<0.05$; Fig. 9C-9F). Similarly, EX $^{\mathrm{miR}-132-3 p}$ exhibited a greater beneficial effect compared with EX ${ }^{\text {Con }}$-treated group and NSC23766

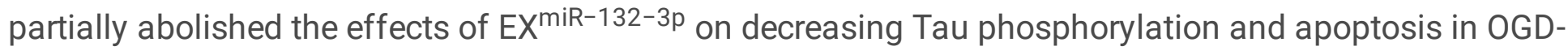
treated neurons (vs EXmiR-132-3p,$p<0.05$; Fig. 9C-9F). These results indicate that miR-132-3p enrichment promotes the effects of MSC EX on reducing Tau hyperphosphorylation and apoptosis in OGD-treated neurons by activating the Ras/Akt/-GSK-3 $\beta$ signaling pathway.

\section{MiR-132-3p mediates EX-induced increasing of neurite outgrowth and synaptic density in OGD-injured neurons by activating the Ras/Akt/-GSK-3 $\beta$ signaling pathway}

To investigate the effect of MSC EX and their carried miR-132-3p on neurite outgrowth in OGD-treated neurons, EXCon / EXmiR-132-3p were co-cultured with OGD-injured neurons for $24 \mathrm{~h}$. The neurite branch number and total neurite length were compromised in OGD-treated neurons, but were ameliorated by

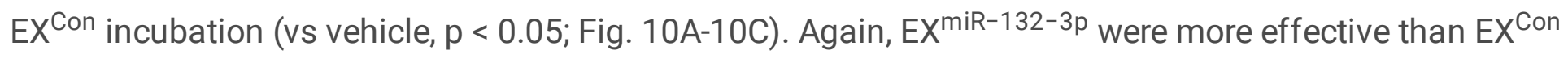
in improving neurite branch number and total neurite length in OGD-injured neurons ( $v s E^{C}{ }^{C o n}, p<0.05$; Fig. 10A-10C). Pretreatment with NSC23766 partially abolished the effects of the EX ${ }^{\text {miR-132-3p }}$ (vs EX ${ }^{\mathrm{miR}-132-3 p}, \mathrm{p}<0.05$; Fig. 10A-10C). 
To determine whether MSC EX and their carried miR-132-3p could promote synaptic density in OGDinjured neurons, we analyzed the expression of synaptophysin (synapsin 1) and postsynaptic density protein (PSD95) by immunofluorescence staining. We observed that EX incubation significantly increased synapsin 1 and PSD95 expression which were compromised in OGD-treated neurons (vs Sham, $p<0.05$; Fig. 10D-10F), and EXmiR-132-3p displayed better efficacy. Furthermore, pretreatment with NSC23766 partially abolished the effects of the EX ${ }^{\mathrm{miR}-132-3 p}$ on increasing synapsin 1 and PSD95 expression in OGD-treated neurons (vs EXmiR-132-3p,$p<0.05$; Fig. 10D-10F).

Taken together, these results indicate that miR-132-3p enrichment enhances the effects of MSC EX on improving neurite outgrowth and synaptic density in OGD-injured neurons.

\section{Discussion}

This study demonstrates that infusion of miR-132-3p-enriched MSC EX improves synaptic and cognitive function in VD mice by increasing miR-132-3p and MSC EX levels, but decreasing A $\beta$ aggregation, Tau hyperphosphorylation, and neuron damage in the cortex and hippocampus. Furthermore, in vitro mechanistic studies have found miR-132-3p priming enhanced the effects of MSC EX on protecting cells from OGD-induced oxidative stress, apoptosis, and synaptic function disruption by targeting RASA1 and activating the downstream Ras/Akt/GSK-3ß signaling pathway in primary cultured neurons.

Ischemia-induced neuron damage as well as dendritic and synaptic abnormalities can lead to neuronal dysfunction, which is the primary cause of cognitive impairment in VD [1]. MSCs are known as "sentinel and safe-guards of injury" and promote neuroprotection in various neurological diseases, including $A D$, ischemic stroke (IS), and vascular cognitive impairment (VCI)[21]. It was found that the neuroprotective effects of MSC depended primarily on exosome-mediated paracrine rather than cell replacement[22]. In this study, we firstly observed that MSC EX levels were significantly decreased in the brain tissue of VD mice, concomitant with cognitive impairment. Additionally, our data showed an impaired endogenous protective effect of MSC EX during VD injury and along with other groups, indicating systemic administration of MSC EX could improve neuronal function in an IS animal model, which is consistent with the findings of other studies $[10,16]$. In the AD mice model, infusion of MSC EX ameliorates learning and memory deficits by decreasing $A \beta$ accumulation and restoring synaptic function [8]. However, to our knowledge, the therapeutic effects of MSC EX in VD are still unknown. Therefore, MSC EX in the present study was systemically administrated to VD mice via the tail vein, which entered the brain and restored MSC EX levels in the hippocampus and cortex explore the specific mechanism of MSC EX in VD mice. Interestingly, MSC EX infusion improved learning and memory in the VD mice, which indicate a therapeutic effect of MSC EX in VD. Since the regulation of neuronal density, spine formation, and synaptic plasticity are critical in maintaining normal cognitive function[23, 24], we here evaluated the effect of MSC EX infusion on neuronal cell density and architecture, spine formation, and synaptic plasticity in the hippocampus and cortex by using H\&E staining and immunofluorescence. As expected, MSC EX infusion rescued VD-induced neuronal cell loss and abnormal architecture[17], spine formation, and synaptic plasticity impairment [25]. Our findings provide new evidence regarding the therapeutic 
efficacy of MSC EX in cognitive decline[8]. However, we do not exclude the possibility that the miR-132-3p enriched MSC EX treatment also merged into cerebral endothelial cells, astrocytes, and microglia in cortex and hippocampus, which also may contribute to protect neuron dysfunction and cognitive impairment from VD.

EX can regulate target cell function by delivering their cargos (miRs, proteins, and lipids) obtained from source cells and miRs are important functional cargos [26]. MiR-132 is one of the most abundant miRs in the brain, where it regulates neuronal function and promotes synaptic plasticity[27]. In addition, a decrease in miR-132 expression was found in postmortem $A D[28]$ and was associated with impaired cognitive function [29]. In the present study, we found that the level of miR-132-3p is obviously decreased in the hippocampus and cortex of VD mice, suggesting that miR-132-3p plays a role in VD; however, little is known about the protective effects of MSC EX enriched with miR-132-3p. Based on this evidence, we transfected MSC with miR-132-3p mimics to generate miR-132-3p-enriched MSC EX, and determined whether EXmiR-132-3p infusion could enhance the therapeutic effects of MSC EX on VD. EXmiR-132-3p infusion significantly increased the level of miR-132-3p in the hippocampus and cortex of VD mice, while EXantagomiR-132-3p decreased cortical and hippocampal miR-132-3p expression compared with EXmiR-132-3p-treatment, suggesting the effective transfer of miR-132-3p to the brain by EXmiR-132-3p . Following intravenous administration, PKH26-labeled MSC EX crosses the blood brain barrier and merges into neurons in the hippocampus and cortex of VD mice. We and others have shown that the systemic administrated MSC EX enriched with neurovascular protective signaling molecules, such as miRs, can be incorporated into neurovascular cells to modify their functions in the brain[6, 16]. Therefore, we further investigated the protective role of MSC EX and their carried miR-132-3p in neuronal dysfunction and cognitive impairment during VD. Our data revealed that the neuronal density, spine formation, and synaptic plasticity in the cortex and hippocampus of VD mice were further increased by the miR-132-3p enriched MSC EX treatment. This is consistent with a previous study, which indicated miR-132 could reduce neuron death, and promote neurite elongation and branching[27]. Taken together, miR-132-3p promotes the therapeutic effects of MSC EX on neuron impairment of VD.

We further investigated the potential mechanisms involved in the therapeutic effects of MSC EX and their miR-132-3p cargo in VD mice. The increased levels of $A \beta$ aggregation and Tau hyperphosphorylation in brain tissue plays an important role in inducing neuron damage, synaptic dysfunction, and cognitive impairment in various neurological disorders $[11,27]$. Evidence shows that MSC EX may decrease both secreted and intracellular $A \beta$ levels in N2a cells[30], and restore the $A \beta$-induced synaptic plasticity dysfunction in the hippocampus of AD mice [7]. In the present study, we demonstrated that MSC EX infusion significantly decreased $A \beta$ levels and accumulation in the cortex and hippocampus of VD mice. Our findings are consistent with a previous report demonstrating that MSC EX protect neurons from synapse damage by promoting A $\beta$ clearance[31]. Moreover, we observed that MSC EX infusion reduced Tau hyperphosphorylation in the cortex and hippocampus of VD mice, which furtherly proved the role of MSC in reducing Tau hyperphosphorylation[32]. Recently, MiR-132-3p was found to play an important role in regulating $A \beta$ production and Tau hyperphosphorylation. Of note, the level of Tau phosphorylation and 
aggregation was markedly increased in miR-132-deficient mice[11]. In the AD mouse model, deletion of miR-132 also promoted $A \beta$ production and plaque accumulation[33]. Likewise, we demonstrated in this study that $E X^{m i R-132-3 p}$ infusion was more effective at decreasing $A \beta$ production, aggregation, and Tau hyperphosphorylation in the cortex and hippocampus in VD mice, suggesting MSC EX and their miR-132$3 p$ cargo exert therapeutic effects on VD by reducing $A \beta$ production and Tau hyperphosphorylation.

GSK-3 $\beta$ is one of the most important protein kinases implicated in the regulation of $A \beta$ production and Tau phosphorylation, and related to neuronal dysfunction and cognitive impairment[34]. Activation of Akt increases phosphorylation of GSK-3 $\beta$ at serine 9 (GSK-3 $\left.\beta^{\text {ser } 9}\right)$ then inhibits the activation of GSK-3 $\beta[35]$, which can enhance synaptic plasticity and cognitive function[34]. Conversely, reducing Akt phosphorylation was associated with GSK-3 $\beta$ activation and ultimately resulted in synaptic plasticity and memory impairment[36]. Thus, Akt/GSK-3 $\beta$ signaling plays a central role in regulating neuronal function and synaptic plasticity. Among the many direct target proteins involved in signaling downstream of miR132-3p, RASA1 mediates the activity of miR-132 in neuronal function and synaptic plasticity[37]. Here, we demonstrated that miR-132-3p enhanced the effects of MSC EX in down-regulating RASA1 and upregulating Ras, and subsequently increasing the phosphorylation of Akt and GSK-3 $\beta^{\text {ser9 }}$, which thereby decreased Tau hyperphosphorylation in OGD-treated neurons. Using a Ras inhibitor, we further demonstrated that MSC EX with miR-132-3p enrichment increase neuron viability and neurite outgrowth, while decreasing neuronal apoptosis in OGD-treated neurons by activating the Ras/Akt/GSK-3 $\beta$ signaling pathway. This is consistent with our in vivo data showing decreased neuronal cell loss and synaptic

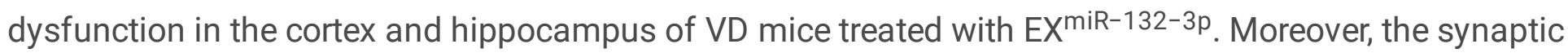
proteins, including synapsin 1 and PSD95 are critical in maintaining normal synaptic function[8]. MSC EX with miR-132-3p enrichment markedly increased synapsin 1 and PSD95 expression by activating the Ras/Akt/GSK-3 $\beta$ signaling pathway in OGD-treated neurons. However, since the potential targets of the miR-132-3p are vast and miR-132-3p-mediated signaling pathways are complex under VD condition. our results on the mechanism of miR-132-3p through RASA1/Ras/Akt/GSK-3 $\beta$ signaling pathway did not to exclude the possibility of its other target of miR-132-3p, such as heterogeneous nuclear ribonucleoprotein $\mathrm{U}$ (HNRNPU)[38], histone deacetylase 3 (HDAC3)[39], and mitogen-activated protein kinase kinase kinase 3 (MEKK3)[40], which may also participate in the enhancement of neuroprotective effect and synaptic plasticity of miR-132-3p enriched MSC EX. To further confirm that the miR-132-3p enriched MSC EX promote neuron protection and synaptic remodeling through the Ras/Akt/ GSK-3 $\beta$ signaling pathway, experiments inviting specific overexpressing RASA1 as well as specific knock down of Ras, and phosphorylation of Akt and GSK-3 $\beta$ in the neurons are needed to be performed in vivo or in vitro.

\section{Conclusions}

In summary, our findings suggest that MSC EX represent a promising therapeutic strategy for VD by ameliorating neuron damage, synaptic dysfunction, and cognitive impairment. Furthermore, they prevent $A \beta$ production, aggregation, and Tau hyperphosphorylation, whereas miR-132-3p enrichment enhances these beneficial effects. Our data further demonstrate that miR-132-3p increases the protective effects of 
MSC EX on OGD-induced neuron apoptosis, synaptic disruption, and Tau hyperphosphorylation through activation of the Ras/Akt/GSK-3 $\beta$ signaling pathway.

\section{Abbreviations}

VD Vascular dementia

MiR-132-3p MicroRNA-132-3p

MSC Mesenchymal stromal cells

EXs Exosomes

OGD Oxygen-glucose deprivation

Rasa $1 \quad$ RAS p21 protein activator 1

p-Akt Phosphorylation-Akt

PBS Phosphate-buffered saline

CNS Central nervous system

NTA Nanoparticle tract analysis

MWM Morris water maze

qRT-PCR Quantitative real-time PCR

NTA Nanoparticle tract analysis

H\&E Hematoxylin and eosin

DHE Dihydroenthidium

PVDF Polyvinylidene difluoride membranes

PSD 95 Postsynaptic density protein 95

\section{Declarations}

\section{Ethics approval and consent to participate}

All animal studies were approved by the Laboratory Animal Care and Use Committees at Guangdong Medical University (Protocol No. GDY1701032). All procedures were performed in accordance with Guangdong Medical University's guidelines. 
Availability of data and materials

All data generated or analyzed during this study are included in this published article.

\section{Funding}

This work was supported by National Natural Science Foundation of China (NSFC, no. 81770500 , 81870580), Guangdong Basic and Applied Basic Research Foundation (2019A1515011574), Finance fund for science and technology special competitive allocation project of Zhanjiang city (no. 2016A01014), "Clinical Medicine" + Science and technology Cooperation Project of affiliated hospital of Guangdong medical university (no. CLP2021B005), PhD initiation Project of affiliated hospital of Guangdong medical university (no. 2021023563).

\section{Authors' contributions}

$X M, Y W, Y S, Q L, J L, Q P$ performed experiments, $X M, Y W, W Z$, QP wrote the manuscript, $X M, Y W, X Y, Q P$ contributed to manuscript preparation, All authors discussed the results, analyzed data and commented on the manuscript, WZ, QP developed the concepts and designed the study. All authors read and approved the final manuscript.

\section{Consent for publication}

Not applicable.

\section{Competing interests}

The authors declare that they have no competing interests.

\section{Author details}

${ }^{1}$ Department of Neurology, Guangdong Key Laboratory of Age-related Cardiac and Cerebral Diseases, Affiliated Hospital of Guangdong Medical University, Zhanjiang 524001, China, ${ }^{2}$ Institute of Biochemistry and Molecular Biology, Guangdong Medical University, Zhanjiang 524001, China.

\section{Acknowledgements}

Not applicable.

\section{References}

1. Iadecola C. The pathobiology of vascular dementia. Neuron. 2013,80:844-66.

2. Yang $Y, X u H Y$, Deng QW, Wu GH, Zeng X, Jin H, et al. Electroacupuncture facilitates the integration of a grafted TrkC-modified mesenchymal stem cell-derived neural network into transected spinal cord in rats via increasing neurotrophin-3. CNS Neurosci Ther. 2021,27:776-91. 
3. Chen JJ, Zhou SH. Mesenchymal stem cells overexpressing MiR-126 enhance ischemic angiogenesis via the AKT/ERK-related pathway. Cardiol J. 2011,18:675-81.

4. Wang L, Qing L, Liu H, Liu N, Qiao J, Cui C, et al. Mesenchymal stromal cells ameliorate oxidative stress-induced islet endothelium apoptosis and functional impairment via Wnt4-beta-catenin signaling. Stem Cell Res Ther. 2017,8:188.

5. Gu Y, He M, Zhou X, Liu J, Hou N, Bin T, et al. Endogenous IL-6 of mesenchymal stem cell improves behavioral outcome of hypoxic-ischemic brain damage neonatal rats by supressing apoptosis in astrocyte. Sci Rep. 2016,6:18587.

6. Xin H, Li Y, Buller B, Katakowski M, Zhang Y, Wang X, et al. Exosome-mediated transfer of miR-133b from multipotent mesenchymal stromal cells to neural cells contributes to neurite outgrowth. Stem Cells. 2012,30:1556-64.

7. Wang SS, Jia J, Wang Z. Mesenchymal Stem Cell-Derived Extracellular Vesicles Suppresses iNOS Expression and Ameliorates Neural Impairment in Alzheimer's Disease Mice. J Alzheimers Dis. 2018,61:1005-13.

8. Cui GH, Wu J, Mou FF, Xie WH, Wang FB, Wang QL, et al. Exosomes derived from hypoxiapreconditioned mesenchymal stromal cells ameliorate cognitive decline by rescuing synaptic dysfunction and regulating inflammatory responses in APP/PS1 mice. FASEB J. 2018,32:654-68.

9. Xin H, Katakowski M, Wang F, Qian JY, Liu XS, Ali MM, et al. MicroRNA cluster miR-17-92 Cluster in Exosomes Enhance Neuroplasticity and Functional Recovery After Stroke in Rats. Stroke. 2017,48:747-53.

10. Wayman GA, Davare M, Ando H, Fortin D, Varlamova O, Cheng HY, et al. An activity-regulated microRNA controls dendritic plasticity by down-regulating p250GAP. Proc Natl Acad Sci U S A. 2008,105:9093-8.

11. Salta E, Sierksma A, Vanden Eynden E, De Strooper B. miR-132 loss de-represses ITPKB and aggravates amyloid and TAU pathology in Alzheimer's brain. EMBO Mol Med. 2016,8:1005-18.

12. Smith PY, Hernandez-Rapp J, Jolivette F, Lecours C, Bisht K, Goupil C, et al. miR-132/212 deficiency impairs tau metabolism and promotes pathological aggregation in vivo. Hum Mol Genet. 2015,24:6721-35.

13. Hancock ML, Preitner N, Quan J, Flanagan JG. MicroRNA-132 is enriched in developing axons, locally regulates Rasa1 mRNA, and promotes axon extension. J Neurosci. 2014,34:66-78.

14. Castaneda P, Munoz M, Garcia-Rojo G, Ulloa JL, Bravo JA, Marquez R, et al. Association of Ncadherin levels and downstream effectors of Rho GTPases with dendritic spine loss induced by chronic stress in rat hippocampal neurons. J Neurosci Res. 2015,93:1476-91.

15. Hira K, Ueno Y, Tanaka R, Miyamoto N, Yamashiro K, Inaba T, et al. Astrocyte-Derived Exosomes Treated With a Semaphorin 3A Inhibitor Enhance Stroke Recovery via Prostaglandin D2 Synthase. Stroke. 2018,49:2483-94.

16. Pan Q, Kuang X, Cai S, Wang X, Du D, Wang J, et al. miR-132-3p priming enhances the effects of mesenchymal stromal cell-derived exosomes on ameliorating brain ischemic injury. Stem Cell Res 
Ther. 2020,11:260.

17. Siracusa R, Impellizzeri D, Cordaro M, Crupi R, Esposito E, Petrosino S, et al. Anti-Inflammatory and Neuroprotective Effects of Co-UltraPEALut in a Mouse Model of Vascular Dementia. Front Neurol. 2017,8:233.

18. Xin H, Li Y, Cui Y, Yang JJ, Zhang ZG, Chopp M. Systemic administration of exosomes released from mesenchymal stromal cells promote functional recovery and neurovascular plasticity after stroke in rats. J Cereb Blood Flow Metab. 2013,33:1711-5.

19. Perez-Gonzalez R, Gauthier SA, Kumar A, Saito M, Saito M, Levy E. A Method for Isolation of Extracellular Vesicles and Characterization of Exosomes from Brain Extracellular Space. Methods Mol Biol. 2017,1545:139-51.

20. Shelat PB, Plant LD, Wang JC, Lee E, Marks JD. The membrane-active tri-block copolymer pluronic F68 profoundly rescues rat hippocampal neurons from oxygen-glucose deprivation-induced death through early inhibition of apoptosis. J Neurosci. 2013,33:12287-99.

21. Lee NK, Kim H, Chang JW, Jang H, Kim H, Yang J, et al. Exploring the Potential of Mesenchymal Stem Cell-Based Therapy in Mouse Models of Vascular Cognitive Impairment. Int J Mol Sci. 2020,21.

22. Corrigendum to: Concise Review: MSC-Derived Exosomes for Cell-Free Therapy. Stem Cells. 2017,35:2103.

23. Ho VM, Lee JA, Martin KC. The cell biology of synaptic plasticity. Science. 2011,334:623-8.

24. Kazim SF, Iqbal K. Neurotrophic factor small-molecule mimetics mediated neuroregeneration and synaptic repair: emerging therapeutic modality for Alzheimer's disease. Mol Neurodegener. 2016,11:50.

25. Yu P, Venkat P, Chopp M, Zacharek A, Shen Y, Ning R, et al. Role of microRNA-126 in vascular cognitive impairment in mice. J Cereb Blood Flow Metab. 2019,39:2497-511.

26. Fang SB, Zhang HY, Wang C, He BX, Liu XQ, Meng XC, et al. Small extracellular vesicles derived from human mesenchymal stromal cells prevent group 2 innate lymphoid cell-dominant allergic airway inflammation through delivery of miR-146a-5p. J Extracell Vesicles. 2020,9:1723260.

27. El Fatimy R, Li S, Chen Z, Mushannen T, Gongala S, Wei Z, et al. MicroRNA-132 provides neuroprotection for tauopathies via multiple signaling pathways. Acta Neuropathol. 2018,136:53755.

28. Lau P, Bossers K, Janky R, Salta E, Frigerio CS, Barbash S, et al. Alteration of the microRNA network during the progression of Alzheimer's disease. EMBO Mol Med. 2013,5:1613-34.

29. Patrick E, Rajagopal S, Wong HA, McCabe C, Xu J, Tang A, et al. Dissecting the role of non-coding RNAs in the accumulation of amyloid and tau neuropathologies in Alzheimer's disease. Mol Neurodegener. 2017,12:51.

30. An K, Klyubin I, Kim Y, Jung JH, Mably AJ, O'Dowd ST, et al. Exosomes neutralize synaptic-plasticitydisrupting activity of Abeta assemblies in vivo. Mol Brain. 2013,6:47. 
31. de Godoy MA, Saraiva LM, de Carvalho LRP, Vasconcelos-Dos-Santos A, Beiral HJV, Ramos AB, et al. Mesenchymal stem cells and cell-derived extracellular vesicles protect hippocampal neurons from oxidative stress and synapse damage induced by amyloid-beta oligomers. J Biol Chem. 2018,293:1957-75.

32. Lee HJ, Lee JK, Lee H, Shin JW, Carter JE, Sakamoto T, et al. The therapeutic potential of human umbilical cord blood-derived mesenchymal stem cells in Alzheimer's disease. Neurosci Lett. 2010,481:30-5.

33. Hernandez-Rapp J, Rainone S, Goupil C, Dorval V, Smith PY, Saint-Pierre M, et al. microRNA-132/212 deficiency enhances Abeta production and senile plaque deposition in Alzheimer's disease triple transgenic mice. Sci Rep. 2016,6:30953.

34. Ali T, Kim MO. Melatonin ameliorates amyloid beta-induced memory deficits, tau hyperphosphorylation and neurodegeneration via PI3/Akt/GSk3beta pathway in the mouse hippocampus. J Pineal Res. 2015,59:47-59.

35. Manning BD, Cantley LC. AKT/PKB signaling: navigating downstream. Cell. 2007,129:1261-74.

36. Ma T, Du X, Pick JE, Sui G, Brownlee M, Klann E. Glucagon-like peptide-1 cleavage product GLP-1(936) amide rescues synaptic plasticity and memory deficits in Alzheimer's disease model mice. $J$ Neurosci. 2012,32:13701-8.

37. Wanet A, Tacheny A, Arnould T, Renard P. miR-212/132 expression and functions: within and beyond the neuronal compartment. Nucleic Acids Res. 2012,40:4742-53.

38. Qu J, Xiong X, Hujie G, Ren J, Yan L, Ma L. MicroRNA-132-3p alleviates neuron apoptosis and impairments of learning and memory abilities in Alzheimer's disease by downregulation of HNRNPU stabilized BACE1. Cell Cycle. 2021:1-12.

39. Zeng C, Meng X, Mai D, Xu K, Qu S. Overexpression of miR-132-3p contributes to neuronal protection in in vitro and in vivo models of Alzheimer's disease. Behav Brain Res. 2021,417:113584.

40. Fang H, Li HF, Pan Q, Jin HL, Yang M, Wang RR, et al. MiR-132-3p Modulates MEKK3-Dependent NFkappaB and p38/JNK Signaling Pathways to Alleviate Spinal Cord Ischemia-Reperfusion Injury by Hindering M1 Polarization of Macrophages. Front Cell Dev Biol. 2021,9:570451.

\section{Figures}

\section{Figure 1}

EXmiR-132-3p infusion restores cortical and hippocampal MSC EX and miR-132-3p level in VD mice. (A-C) MSC EX from sham and VD mouse brain tissue were detected by NTA, TEM, and western blotting. (D) The effects of EXCon/ EXmiR-132-3p/ EXantagomiR-132-3p infusion on cortical and hippocampal MSC EX level in Sham and VD mice. (E) The effects of EXCon /EXmiR-132-3p /EXantagomiR-132-3p infusion on 
cortical and hippocampal miR-132-3p level in Sham and VD mice. Data represent the mean $\pm S E M, n=8$ mice per group. * $\mathrm{p} \otimes 0.05$.

\section{Figure 2}

EXmiR-132-3p infusion rescues cognitive impairment and synaptic deficits in VD mice. (A) Workflow of MSC EX injection / rescue experiments. (B) Represent images showing that injected EX (PKH 26, red) merged into cortical and hippocampal neurons (NeuN, green). (C-F) The effects of EX injection on cognitive impairment in VD mice in MWM tests. Data represent the mean \pm SEM. Three independent experiments were performed ( $n=3$ mice per group). * $\mathrm{p} \otimes 0.05$.

\section{Figure 3}

EXmiR-132-3p infusion rescues synaptic deficits in VD mice. (A) Representative Golgi staining from cortex and hippocampus regions are shown. Scale bar, $10 \mu \mathrm{m}$. (B) The effects of MSC EX injection on cortical dendritic arborization in Sham and VD mice. (C-E) Representative images and summary data of dendritic spines from cortical and hippocampal neurons from VD mice after treated with EXCon / EXmiR132-3p/ EXantagomiR-132-3p. Data represent the mean \pm SEM. $n=20$ neurons from 3 mice per group. Three independent experiments were performed. *p囚0.05.

\section{Figure 4}

EXmiR-132-3p infusion reduces cortical neuron loss and abnormal architecture in hippocampal CA1 region in VD mice, injected with various MSC EX. $(A, C)$ The cortical neuron number in Sham and VD mice were detected by immunofluorescence staining (NeuN, green, DAPI, blue). (B, D) Representative images and summary data showing the hippocampal neuron number and architecture in VD mice by immunochemistry. Data represent the mean \pm SEM. $n=20$ fields from 3 mice per group. Three independent experiments were performed. ${ }^{*} \mathrm{p} \otimes 0.05$.

\section{Figure 5}

EXmiR-132-3p infusion reduces cortical neuron apoptosis in VD mice. The apoptotic neurons in cortex were detected by immunofluorescence staining (TUNEL, red, NeuN, green). Scale bar, $30 \mu \mathrm{m}$. Data represent the mean \pm SEM. $n=20$ fields from 3 mice per group. Three independent experiments were performed. ${ }^{\star} \mathrm{p} \otimes 0.05$.

\section{Figure 6}


EXmiR-132-3p infusion reduces cortical and hippocampal $A \beta$ production in VD mice. $(A, C)$ The $A \beta$ production in cortex was detected by immunofluorescence staining (A $\beta$, red, MAP-2, green). Scale bar, 40 $\mu \mathrm{m}$. $(B, D)$ The $A \beta$ production in hippocampus was detected by immunofluorescence staining $(A \beta$, red, MAP-2, green). Scale bar, $40 \mu \mathrm{m}$. Data represent the mean \pm SEM. $n=20$ fields from 3 mice per group. Three independent experiments were performed. * $₫ 0.05$.

\section{Figure 7}

EXmiR-132-3p infusion reduces cortical and hippocampal Tau hyperphosphorylation in VD mice. (A, C) The Tau hyperphosphorylation in cortex was detected by immunofluorescence staining ( $p$-Tau, red, MAP2, green). Scale bar, $40 \mu \mathrm{m}$. (B, D) The Tau hyperphosphorylation in hippocampus was detected by immunofluorescence staining ( $\mathrm{p}-\mathrm{Tau}$, red, MAP-2, green). Scale bar, $40 \mu \mathrm{m}$. Data represent the mean \pm SEM. $\mathrm{n}=20$ fields from 3 mice per group. Three independent experiments were performed. ${ }^{\star} \mathrm{p} \otimes 0.05$.

\section{Figure 8}

EXmiR-132-3p incubation increases miR-132-3p level, and down-regulated the target protein RASA1 expression in OGD-treated neurons. (A) Immunofluorescence of MSC EX merged with neurons after incubation (MSC EX, red, $\beta 111$-tubulin, green). Scale bar, $20 \mu \mathrm{m}$. (B) MiR-132-3p level in OGD-treated neurons were measured by qRT-PCR. (C) miR-132-3p regulates the target protein RASA1 expression in OGD-treated neurons. Data represent the mean \pm SEM. Three independent experiments were performed. *p $₫ 0.05$.

\section{Figure 9}

EXmiR-132-3p incubation decreasing neuron apoptosis and tau phosphorylation by activating Ras/ Akt/ GSK-3 $\beta$ signaling pathway in OGD-treated neurons. (A, B) Representative images and summary data showing the level of Ras and phosphorylation of Akt and GSK-3 $\beta$ in OGD-treated neurons after various MSC EX incubation. (C) The neuron apoptosis was detected by flow cytometry. (D, E) Representative images and summary data showing the p-Tau level in OGD-treated neurons after various MSC EX incubation (p-Tau, green, nucleus, blue). Scale bar, $40 \mu \mathrm{m}$. (F) The phosphorylation of Tau in OGD-treated neurons after various MSC EX incubation were measured by western blotting. Data represent the mean \pm SEM. Three independent experiments were performed. *p®0.05.

\section{Figure 10}

EXmiR-132-3p incubation restores neurite outgrowth and synaptic density in OGD-treated neurons. (A-C) Representative images and summary data showing neurite outgrowth in OGD-treated neurons after 
various MSC EX incubation ( $\beta 111$-tubulin, green, nucleus, blue). Scale bar, $30 \mu \mathrm{m}$. (D-F) Representative images and summary data showing synaptic density in OGD-treated neurons after various MSC EX incubation (PSD95, red, synaptophysin, green). Data represent the mean \pm SEM. Three independent experiments were performed. ${ }^{*} \mathrm{p} \varangle 0.05$. 